\title{
A contemporary analysis of pulmonary hypertension in patients undergoing mitral valve surgery: Is this a risk factor?
}

\author{
Daniel H. Enter, MD, ${ }^{\mathrm{a}}$ Anthony Zaki, BS, ${ }^{\mathrm{a}}$ Brett F. Duncan, MD, ${ }^{\mathrm{a}}$ Jane Kruse, RN, BSN, \\ Adin-Cristian Andrei, PhD, ${ }^{a} \mathrm{Zhi} \mathrm{Li}, \mathrm{MS},{ }^{\mathrm{a}} \mathrm{S}$. Chris Malaisrie, MD, ${ }^{\mathrm{a}}$ Sanjiv J. Shah, MD, \\ James D. Thomas, MD, ${ }^{b}$ and Patrick M. McCarthy, MD ${ }^{\mathrm{a}}$
}

\begin{abstract}
Objective: Pulmonary hypertension (PHT) has been considered a risk factor for mortality in cardiac surgery. Among mitral valve surgery (MVS) patients, we sought to determine if severe PHT increases mortality risk and if patients who undergo concomitant tricuspid valve surgery (TVS) incur additional risk.
\end{abstract}

Methods: Preoperative PHT was assessed in 1571 patients undergoing MVS, from 2004 to 2013. Patients were stratified into PHT groups as follows (mm $\mathrm{Hg}$ ): none $(<35)$; moderate (35-49); severe (50-79); and extreme $(\geq 80)$. Propensity-score matching resulted in a total of 430 patients, by PHT groups, and 384 patients, by TVS groups.

Results: Patients with severe PHT had higher mortality, both 30-day (4\% PHT vs $1 \%$ no PHT, $P<.02$ ) and late (defined as survival at 5 years): $75.5 \%$ severe versus $91.9 \%$ no PHT $(P<.001)$. In propensity-score-matched groups, severe PHT was not a risk factor for 30 -day ( $3 \%$ each, $P=1.0)$ or late mortality $(86.2 \%$ severe vs $87.1 \%$ no PHT; $P=.87$ ). TVS did not increase 30 -day ( $4.7 \%$ TVS vs $4.2 \%$ no TVS, $P=.8)$ or late mortality $(78.7 \%$ TVS vs $75.3 \%$ no TVS, $P=.90)$. Late survival was lower in extreme PHT (75.4\% vs no PHT 91.5\%, $P=.007)$, and a trend was found in 30-day mortality ( $11 \%$ extreme vs $3 \%$ no PHT, $P=.16)$.

Conclusions: Mortality in MVS is unaffected by severe PHT or the addition of TVS, yet extreme PHT remains a risk factor. Severe PHT (50-79 mm Hg) should not preclude surgery; concomitant TVS does not increase mortality. (J Thorac Cardiovasc Surg 2016;151:1288-99)

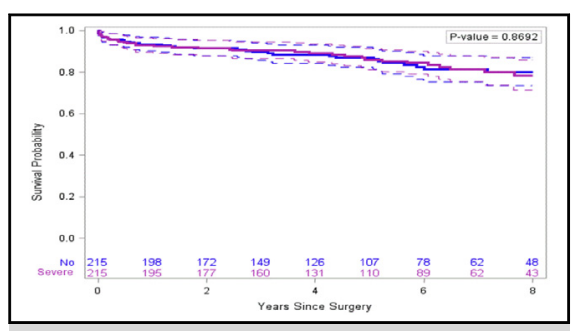

Kaplan-Meier estimates in propensity-score matched groups: severe group (Severe) versus no PHT (No).

\section{Central Message}

Mortality in mitral valve surgery is unaffected by severe pulmonary hypertension or the addition of tricuspid valve surgery, but extreme pulmonary hypertension remains a risk factor.

\section{Perspective}

Pulmonary hypertension is considered a risk factor for 30-day mortality in MVS patients. Our study found no difference in mortality for MVS patients with severe PHT or with the addition of TVS. Severe PHT should not be a contraindication to MVS, and TVS can be safely added; however, extreme PHT remains a consideration when determining operative risk.

See Editorial Commentary page 1300.
Pulmonary hypertension (PHT) historically has been considered a mortality risk factor in cardiac surgical patients, and is found in $15 \%$ to $60 \%$ of patients who have valvular heart disease. PHT is associated with a

From the Divisions of a Cardiac Surgery and ${ }^{\mathrm{b}}$ Cardiology, Bluhm Cardiovascular Institute, Northwestern University Feinberg School of Medicine, Chicago, Ill.

Read at the 95th Annual Meeting of The American Association for Thoracic Surgery, Seattle, Washington, April 25-29, 2015.

Received for publication April 29, 2015; revisions received Dec 18, 2015; accepted for publication Dec 30, 2015; available ahead of print Feb 28, 2016.

Address for reprints: Patrick M. McCarthy, MD, Executive Director of the Bluhm Cardiovascular Institute, Chief of the Cardiac Surgery Division, Heller-Sacks Professor of Surgery at the Northwestern University Feinberg School of Medicine, 201 E Huron St, Ste 11-140, Chicago, IL 60611-2908 (E-mail: pmccart@nm.org). $0022-5223 / \$ 36.00$

Copyright (c) 2016 by The American Association for Thoracic Surgery

http://dx.doi.org/10.1016/j.jtcvs.2015.12.063 higher risk of cardiovascular events with medical management, during valve surgery, and even after successful surgical intervention. ${ }^{1}$ In patients who have mitral valve disease, PHT is a common finding in the preoperative evaluation, ${ }^{2}$ often resulting from elevated left atrial pressures that lead to pulmonary vascular remodeling. ${ }^{3}$ Longstanding PHT increases the afterload on the right ventricle, leading to hypertrophy and eventually, cor pulmonale. ${ }^{3}$ This right-heart

Scanning this $\mathrm{QR}$ code will take you to the article title page. To view the AATS 2015 Webcast, see the URL at the end of the article.

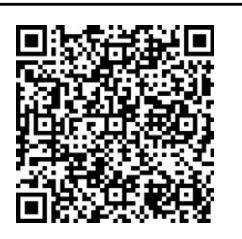




\author{
Abbreviations and Acronyms \\ MVS $=$ mitral valve surgery \\ PASP $=$ pulmonary artery systolic pressure \\ PHT $=$ pulmonary hypertension \\ RHC $=$ right-heart catheterization \\ STS $=$ Society of Thoracic Surgeons \\ TVS $=$ tricuspid valve surgery
}

failure is associated with tricuspid annulus, right ventricular dilation, and tricuspid regurgitation, further exacerbating right ventricular dysfunction. ${ }^{4,5}$ Current guidelines suggest that the most effective therapy for severe degenerative mitral regurgitation is surgical intervention, ${ }^{6}$ yet in some cases, operative treatment is not pursued, owing to perceived high perioperative risks.

In this study, we aim to elucidate the isolated impact of PHT on short- and long-term outcomes of patients who have PHT. Although conventions vary, we defined PHT as the presence of a pulmonary artery systolic pressure (PASP) $>35 \mathrm{~mm} \mathrm{Hg}$, which we have further stratified into moderate $(35-49 \mathrm{~mm} \mathrm{Hg}$ ), severe $(50-79 \mathrm{~mm} \mathrm{Hg})$, and extreme $(\geq 80 \mathrm{~mm} \mathrm{Hg})$. Preoperatively, PASP is either measured directly via right-heart catheterization (RHC), or estimated via Doppler echocardiography, using the simplified Bernoulli equation. ${ }^{7,8}$

Up to $50 \%$ of patients undergoing MVS have tricuspid valve regurgitation, ${ }^{9,10}$ which has been shown to negatively affect survival independently. ${ }^{11}$ Current guidelines provide a Class I indication for repair of severe tricuspid regurgitation for patients undergoing left-sided valve surgery, ${ }^{6}$ consistent with long-term outcomes demonstrating a survival advantage of tricuspid valve repair concomitant with MVS..$^{10,12-14}$ We performed a subset analysis on the MVS patients who had PHT, to assess the impact of concomitant tricuspid valve surgery (TVS) on patient outcomes.

\section{METHODS}

\section{Patients and Study Design}

Data for this project were obtained from the Cardiovascular Research Database in the Clinical Trial Unit of the Bluhm Cardiovascular Institute at Northwestern Memorial Hospital. This database was approved by the Institutional Review Board at Northwestern University. Any subjects who declined to participate in the project were not included in the analysis. Between April 1, 2004, and December 31, 2013, a total of 3755 adult patients underwent first-time valve surgery, excluding patients who had transcatheter aortic valve replacement, ventricular assist devices, transplant, trauma, or endocarditis. Of these, 1714 patients had MVS. Preoperative PASP data were available in 1571 (92\%) cases: 1075 $(62.7 \%$ ) patients had PHT (defined as PASP > $35 \mathrm{~mm} \mathrm{Hg}) ; 496(28.9 \%)$ patients had no PHT; and 143 (8.34\%) patients had unknown PASP values. PHT patients were further stratified into groups based on PASP $(\mathrm{mm} \mathrm{Hg})$ : $600(55.8 \%)$ moderate (35-49); $426(39.6 \%)$ severe (50-79); and 49 $(4.6 \%)$ extreme $(\geq 80)$.
Our institutional database did not indicate whether the PASP was obtained by echocardiography or RHC. In patients who did not receive an RHC study preoperatively, PASP values were obtained from Doppler echocardiography, using the modified Bernoulli equation $(4 \times \text { [tricuspid regurgitation }]^{2}+$ right atrial pressure $) .{ }^{15}$ The absence of pulmonary stenosis and right ventricular outflow obstruction allowed for the estimation of PASP from the right ventricular systolic pressures obtained from echocardiography. When both RHC and echocardiography were obtained preoperatively, results obtained from RHC were used preferentially. No intraoperative echocardiogram measurements were used. Baseline PASP data were not available on 143 patients, who were excluded from this study.

\section{Mortality Data Collection}

Mortality data were aggregated continuously and in an equally thorough manner by a dedicated team of research personnel upon consulting various sources, including the following: (1) the local cardiovascular research database registry, which captures extensive information via postal surveys mailed (to patients alive at discharge) at 6 and 12 months after surgery, and annually thereafter or until notice of death; (2) copies of records for medical procedures and hospitalizations, to verify patient self-reported events captured via survey questionnaires; (3) reviews of external medical records and written or electronic correspondence with the referring or treating physician; (4) direct interviews with the patient during follow-up visits and clinical evaluation; (5) online death indexes, including the Social Security Death Index and genealogy resources (eg, ancestry.com) that provide copies of the death information, for this index, that is supplied directly by family members (at least once a year, our dedicated team reviews the entire cohort of patients known to be alive at last follow-up); and (6) local newspaper death notices, because a substantial segment of our patient population resides in the metropolitan Chicago area or in a state neighboring Illinois. Utilizing these sources, follow-up information was available on $100 \%$ of the cohort. The Society of Thoracic Surgeons (STS) database definitions were used for 30-day mortality.

\section{Statistical Analysis}

Given the risk for confounding due to baseline imbalances, we employed propensity-score matching methods. Groups compared were matched 1-to-1, based on this score, using a greedy algorithm with a caliper of size 0.2 logit propensity-score standard deviation units. The adequacy of between-groups balance in each baseline characteristic used to create the propensity-score model was assessed using standardized differences ${ }^{16}$ (orange color for the prematching groups; violet color for the matched groups). Variables used in the matching process were as follows: age; gender; body surface area; preoperative creatinine level; Ambler score; angina; coronary artery disease; family history of coronary artery disease; diabetes; hypercholesterolemia; hypertension; chronic obstructive pulmonary disease; cerebrovascular accident; prior coronary artery bypass graft; prior valve surgery; repeat sternotomy; prior myocardial infarction; New York Heart Association functional class III or IV; history of atrial fibrillation; elective status; and mitral valve functional class and TVS (except for the subgroup analysis comparing concomitant TVS vs no TVS among MVS patients).

Additional propensity-score matched analyses have compared the incidence of receiving a predischarge permanent pacemaker by concomitant tricuspid valve repair or replacement status, among the subgroup of patients with preoperative PHT, and separately in the entire cohort (PHT classification was included as a variable in the respective propensity-score models). Propensity-score matching was implemented using the SAS macro \% GMATCH (SAS Institute, Inc, Cary, NC).

Kaplan-Meier curves with corresponding $95 \%$ pointwise confidence intervals were used to summarize postoperative overall survival; group comparisons were based on the log-rank test. The association between long-term mortality and PASP as a continuous variable was assessed using 
TABLE 1. Patient characteristics by PHT classification, preoperatively

\begin{tabular}{|c|c|c|c|c|c|}
\hline \multirow[b]{2}{*}{ Variable } & \multirow[b]{2}{*}{ None $(n=496)$} & \multicolumn{3}{|c|}{ PHT (mm Hg) } & \multirow[b]{2}{*}{$P$ value } \\
\hline & & $\begin{array}{c}\text { Moderate (35-49) } \\
(n=600)\end{array}$ & $\begin{array}{l}\text { Severe (50-79) } \\
\quad(n=426)\end{array}$ & $\begin{array}{c}\text { Extreme }(\geq 80) \\
(n=49)\end{array}$ & \\
\hline Age $(y)$, mean \pm SD & $59.4 \pm 13.9$ & $65 \pm 12.9$ & $68 \pm 12.1$ & $65.7 \pm 13.1$ & $<.001$ \\
\hline LV ejection fraction $\%$ & $60(53,65)$ & $57(45,61.5)$ & $55(43,63)$ & $60(53,65)$ & $<.001$ \\
\hline Female gender & $202(41)$ & $279(47)$ & $213(50)$ & $29(59)$ & .008 \\
\hline Diabetes & $48(10)$ & $76(13)$ & $95(22)$ & $12(24)$ & $<.001$ \\
\hline Hypertension & $262(53)$ & $354(59)$ & $282(66)$ & $32(65)$ & $<.001$ \\
\hline Chronic lung disease & $48(10)$ & $86(14)$ & $74(17)$ & $10(20)$ & .004 \\
\hline Cerebrovascular disease & $40(8)$ & $63(11)$ & $62(15)$ & $5(10)$ & .017 \\
\hline Prior stroke & $22(4)$ & $36(6)$ & $33(8)$ & $5(10)$ & .12 \\
\hline Previous MI & $48(10)$ & $78(13)$ & $70(16)$ & 7 (14) & .025 \\
\hline Prior pacemaker & $22(4)$ & $38(6)$ & $46(11)$ & $5(10)$ & .001 \\
\hline Congestive heart failure & $137(28)$ & $246(41)$ & $241(57)$ & $30(61)$ & $<.001$ \\
\hline Mitral stenosis & & & & & $<.001$ \\
\hline Yes & $33(6.5)$ & $81(13.5)$ & $110(26)$ & $20(41)$ & \\
\hline No & $430(87)$ & $471(78.5)$ & $275(64)$ & $24(49)$ & \\
\hline Missing & $33(6.5)$ & $48(8)$ & $41(10)$ & $5(10)$ & \\
\hline NYHA functional class & & & & & $<.001$ \\
\hline I & $157(32)$ & $128(21)$ & $60(14)$ & $2(4)$ & \\
\hline II & $219(44)$ & $234(39)$ & $127(30)$ & $14(29)$ & \\
\hline III & $95(19)$ & $184(31)$ & $188(44)$ & $24(49)$ & \\
\hline IV & $17(3)$ & $49(8)$ & $48(11)$ & $8(16)$ & \\
\hline Missing & $8(1)$ & $5(1)$ & $3(1)$ & $1(2)$ & \\
\hline Coronary artery disease & & & & & $<.001$ \\
\hline Yes & $136(27)$ & $223(37)$ & $192(45)$ & $23(47)$ & \\
\hline No & $346(70)$ & $374(62.5)$ & $230(54)$ & $26(53)$ & \\
\hline Missing & $14(3)$ & $3(0.5)$ & $4(1)$ & $0(0)$ & \\
\hline
\end{tabular}

Values are n (\%), unless otherwise indicated. PHT, Pulmonary hypertension; $S D$, standard deviation; $L V$, left ventricular; $M I$, myocardial infarction; $N Y H A$, New York Heart Association.

unadjusted and covariate-adjusted Cox regression models, with a smoothing spline effect for PASP, ${ }^{17}$ using the $\mathrm{R}$ package smoothHR (cran.r-project.org/package $=$ smoothHR). Variables included in the adjusted model were determined using stepwise selection in a separate Cox regression model; the initial pool of variables consisted of those factors used to create the propensity-score model described earlier. To ensure internal mortality data consistency, we have compared overall survival Kaplan-Meier estimates, by surgery year in the entire cohort, and separately within each pulmonary hypertension severity degree.

Means \pm standard deviations or medians (first quartile, third quartile) were used to summarize continuously distributed variables; the 2-sample unequal variance $t$ test or the Wilcoxon rank-sum test were used to compare groups. Counts and percentages were used to summarize variables that had discrete distributions; $\chi^{2}$ analysis, Kruskal-Wallis analysis of variance, or Fisher exact tests were used to compare groups. Baseline and follow-up PASP data were compared using the paired $t$ test. Tests were 2-sided, without multiplicity adjustments. Statistical analyses were performed using SAS, version 9.3 software (SAS Institute, Inc, Cary, NC), R v. 3.2.1 (www.R-project.org), and RStudio (www.rstudio.com).

\section{RESULTS}

\section{Prematching Groups}

The PHT patients were older and presented with more comorbidities, including prior myocardial infarction, coronary artery disease, cerebrovascular disease, diabetes, congestive heart failure, chronic lung disease, and higher New York Heart Association functional class. Overall,
392 (25\% of 1571$)$ patients had concomitant TVS $(3.6 \%$ replacement; $96.4 \%$ repair). In addition, 244 of the 1571 MVS patients had mitral stenosis, with the highest proportion in the extreme PHT group (20 of 49 [40.8\%]) (Table 1).

\section{After Propensity-Score Matching}

The patients who had PHT had a longer perfusion and crossclamp time, along with more concomitant procedures, including coronary artery bypass grafting, aortic valve surgery, and TVS (Table 2). Mitral valve repair rates were higher among patients who did not have PHT. Those who did have PHT additionally had a longer postoperative length of stay, higher 30-day readmission rates, higher 30-day mortality, and lower long-term overall survival (Figure 1; $P<.001$ ).

Severe versus no PHT. Propensity-score matching resulted in 215 pairs of patients with severe PHT versus no PHT, with adequate baseline covariate balancing (Figure 2, A). Thirty-day mortality (3\% in each group, $P=1.0)$ and 5-year overall survival $(P=.87$; Figure 2 , $B$; 5-year survival: $86.2 \%$ severe PHT vs $87.1 \%$ no PHT) were not significantly different. Postoperative hospital length of stay was similar (median 7 days, $P=.36$ ), as 
TABLE 2. Intraoperative characteristics and postoperative outcomes, by preoperative PHT classification

\begin{tabular}{|c|c|c|c|c|c|}
\hline \multirow[b]{2}{*}{ Variable } & \multirow[b]{2}{*}{ None $(n=496)$} & \multicolumn{3}{|c|}{ PHT } & \multirow[b]{2}{*}{$P$ value } \\
\hline & & $\begin{array}{c}\text { Moderate (35-49) } \\
\quad(n=600)\end{array}$ & $\begin{array}{l}\text { Severe (50-79) } \\
\quad(\mathrm{n}=\mathbf{4 2 6})\end{array}$ & $\begin{array}{l}\text { Extreme }(\geq 80) \\
(n=49)\end{array}$ & \\
\hline Perfusion time (min) & $97(77,129)$ & $117(88,151.5)$ & $128.5(103,162)$ & $140(116,182)$ & $<.001$ \\
\hline Crossclamp time (min) & $80(66,105)$ & $91(69,120)$ & $93(73,121)$ & $107(85,141)$ & $<.001$ \\
\hline Coronary artery bypass graft & $118(24)$ & $178(30)$ & $139(33)$ & $16(33)$ & .021 \\
\hline Aortic valve surgery & $73(15)$ & $135(23)$ & $118(28)$ & $17(35)$ & $<.001$ \\
\hline Tricuspid valve surgery & $42(8)$ & $138(23)$ & $189(44)$ & $23(47)$ & $<.001$ \\
\hline Mitral valve surgery type & & & & & $<.001$ \\
\hline Repair & $421(85)$ & $432(72)$ & $239(56)$ & $17(35)$ & \\
\hline Replacement & $75(15)$ & $168(28)$ & $187(44)$ & $32(65)$ & \\
\hline Postoperative length of stay (d) & $6(4,7)$ & $7(5,9)$ & $8(6,10)$ & $9(6,14)$ & $<.001$ \\
\hline Readmission within 30 days & $52(10.5)$ & $69(11.5)$ & $72(16.9)$ & $8(16.3)$ & .008 \\
\hline 30-d mortality $(\%)$ & $6(1.2)$ & $17(2.8)$ & $16(3.8)$ & $6(12)$ & $<.001$ \\
\hline
\end{tabular}

Values are n (\%), unless otherwise indicated. PHT, Pulmonary hypertension.

were 30 -day readmission rates $(17 \%$ severe vs $15 \%$ no PHT, $P=.7)$. Similarly, no difference was found in outcomes for moderate versus no PHT.

Extreme versus no PHT. In a comparison of extreme PHT versus no PHT, propensity-score matching yielded a total of 37 pairs, showing adequate baseline covariate balancing (Figure 3,A). Long-term overall survival was significantly lower among patients who had extreme PHT $(P=.007$; Figure 3, B; 5-year survival: $75.4 \%$ extreme PHT vs $91.5 \%$ no PHT). A trend toward higher 30-day mortality has been observed for patients with extreme PHT $(11 \%$ vs $3 \%, P=.16)$.

\section{Subgroup Analysis Among PHT Patients: \\ Comparison by Concomitant TVS Status}

In the original groups, patients that underwent concomitant TVS were older $(69.3 \pm 11.8$ vs $64.7 \pm 12.8$ years, $P<.001$ ), had a higher median PASP (54 vs $45 \mathrm{~mm} \mathrm{Hg}$, $P<.001$ ), and a higher percentage of those in New York Heart Association functional class III or IV $(55 \%$ vs $43 \%, P<.001)$ than did the patients who did not have TVS. Postoperatively, patients who underwent TVS had a longer length of stay ( 8 vs 7 days, $P<.001$ ) and had lower

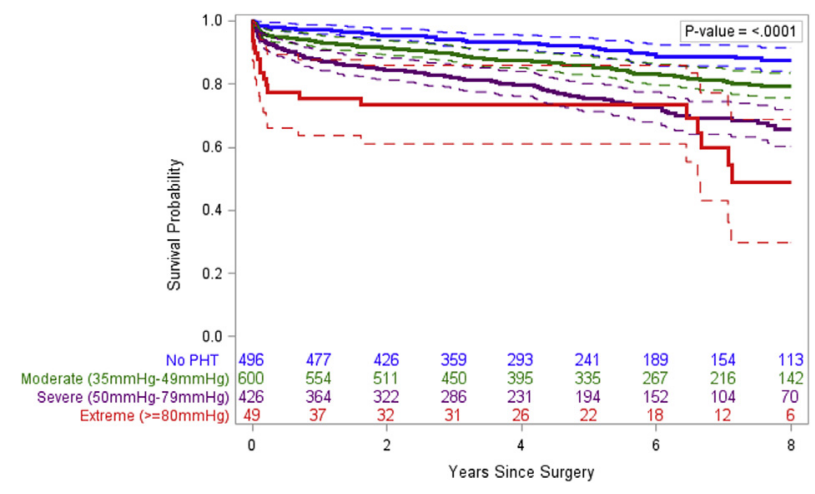

FIGURE 1. Kaplan-Meier survival estimates by pulmonary hypertension (PHT) classification (none, moderate, severe, extreme). long-term overall survival than those with no TVS (5-year survival of $73.7 \%$ vs $84.9 \%$, respectively, $P<.001$ ).

Propensity-score matching yielded 192 pairs of concomitant TVS versus no-TVS patients, with adequate baseline covariate balancing (Figure $4, A$ ). We found no differences in 30 -day mortality ( $5 \%$ for TVS vs $4 \%$ for no TVS, $P=.8)$ or 5 -year overall survival $(P=.90$, 5-year survival: $78.7 \%$ TVS vs $75.3 \%$ no TVS) (Figure 4, B).

\section{Baseline Pulmonary Artery Systolic Pressure as a Continuous Variable}

An exact baseline PASP was available for 1327 of the 1571 study participants. The association between PASP and long-term all-cause mortality in unadjusted and covariate-adjusted (Figure 5, $A$ and $B$ ) models shows that the effect of baseline PASP is nonlinear, and that increased PASP is associated with increased mortality, thus confirming and further refining the unadjusted results (Figure 1). After adjusting for age, Ambler score, creatinine level, chronic obstructive pulmonary disease, and New York Heart Association functional class III or IV, PASP was not a significant risk factor for mortality, except in its high ranges (Figure 5, B). This finding provides further support to our propensity-score matched analyses, which showed that extreme, but not severe or moderate, PHT is associated with increased all-cause mortality. An additional analysis (not shown), adjusting for all the variables included in the propensity-score model, produced results virtually identical to those in Figure 5, B. Internal mortality data consistency checks have revealed that overall Kaplan-Meier survival estimates were not different by surgery year in the entire cohort, or separately within each PHT severity-degree group.

\section{Postsurgical Follow-up}

Among all patients, the mean PASP decreased, from $45.8 \pm 14.6$ to $35.2 \pm 13.1 \mathrm{~mm} \mathrm{Hg}(P<.001)$, with 


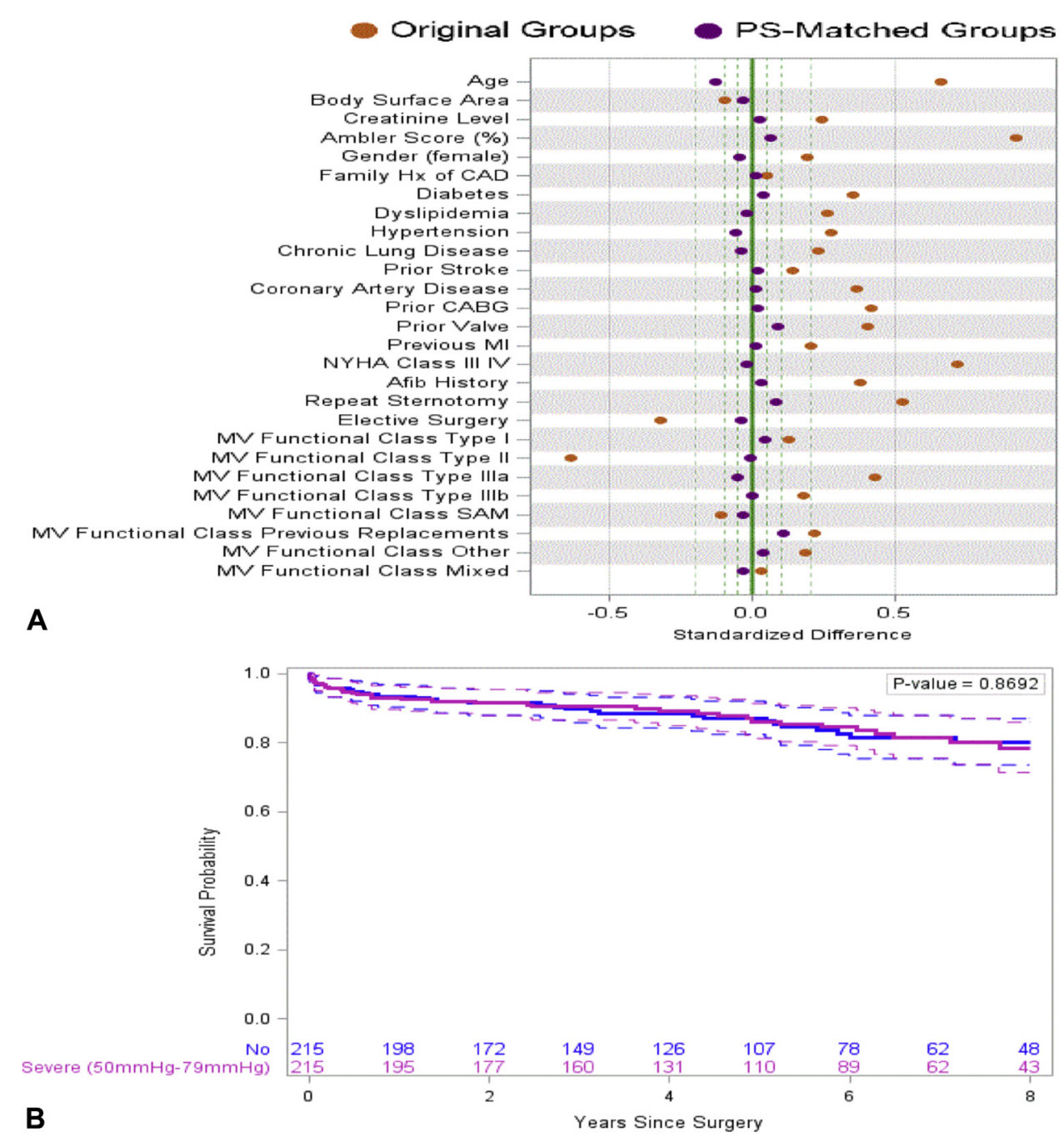

FIGURE 2. Standardized differences in: (A) baseline covariates; and (B) Kaplan-Meier survival estimates in PS-matched analyses for severe versus no PHT. PS, Propensity score; $H x$, history; $C A D$, coronary artery disease; $C A B G$, coronary artery bypass graft; $M I$, myocardial infarction; $N Y H A$, New York Heart Association; Afib, atrial fibrillation; $M V$, mitral valve; $S A M$, systolic anterior motion.

follow-up in $484(31 \%)$ patients during a median of 2 years. The change in the individual PHT groups was as follows $(\mathrm{mm} \mathrm{Hg})$ : extreme $(\mathrm{n}=13)$ : $89.9 \pm 10.5$ to $51.5 \pm 16.1$; severe $(\mathrm{n}=161): 59.2 \pm 7.1$ to $39.2 \pm 14.5$; and moderate $(\mathrm{n}=220): 40.8 \pm 4.5$ to $34 \pm 11.4$; all $P<.001$. For the no-PHT group $(\mathrm{n}=90)$, the decrease was from $28.1 \pm 4.1$ to $28.4 \pm 9.2 \mathrm{~mm} \mathrm{Hg}(P=.7)$.

\section{Incidence of Predischarge Permanent Pacemaker Implantation in Association With Concomitant TVS}

Entire cohort. Patients who underwent concomitant tricuspid valve repair or replacement, compared with those that did not, had a significantly higher incidence of permanent pacemaker implantation prior to discharge (before matching: $12.0 \%$ vs $4.6 \%, P=.001$; propensityscore matching: $12.4 \%$ vs $6.0 \%, P=.031$ ). After excluding discharge deaths and patients that had a permanent pacemaker preoperatively, similar results were observed (before matching: $14.0 \%$ vs $4.9 \%, P=.001$; propensity-score matching: $15.2 \%$ vs $7.9 \%, P=.038)$.

In a comparison of concomitant TVS (repair) versus no TVS, the incidence of permanent pacemaker implantation prior to discharge was significantly different in the beforematching groups $(11.4 \%$ vs $4.6 \%, P=.001)$, but not in the propensity-score matching groups $(10.1 \%$ vs $6.7 \%$, $P=.12$ ). However, after further excluding discharge deaths and those patients that had a permanent pacemaker preoperatively, a higher incidence was observed among concomitant TVS (repair) patients before $(13.7 \%$ vs $4.9 \%, P=.001)$, compared with after $(13.0 \%$ vs $7.6 \%$, $P=.044$ ), matching (Table 3).

PHT patient subgroup. Patients with PHT undergoing concomitant TVS for repair or replacement had a significantly higher incidence of permanent pacemaker 

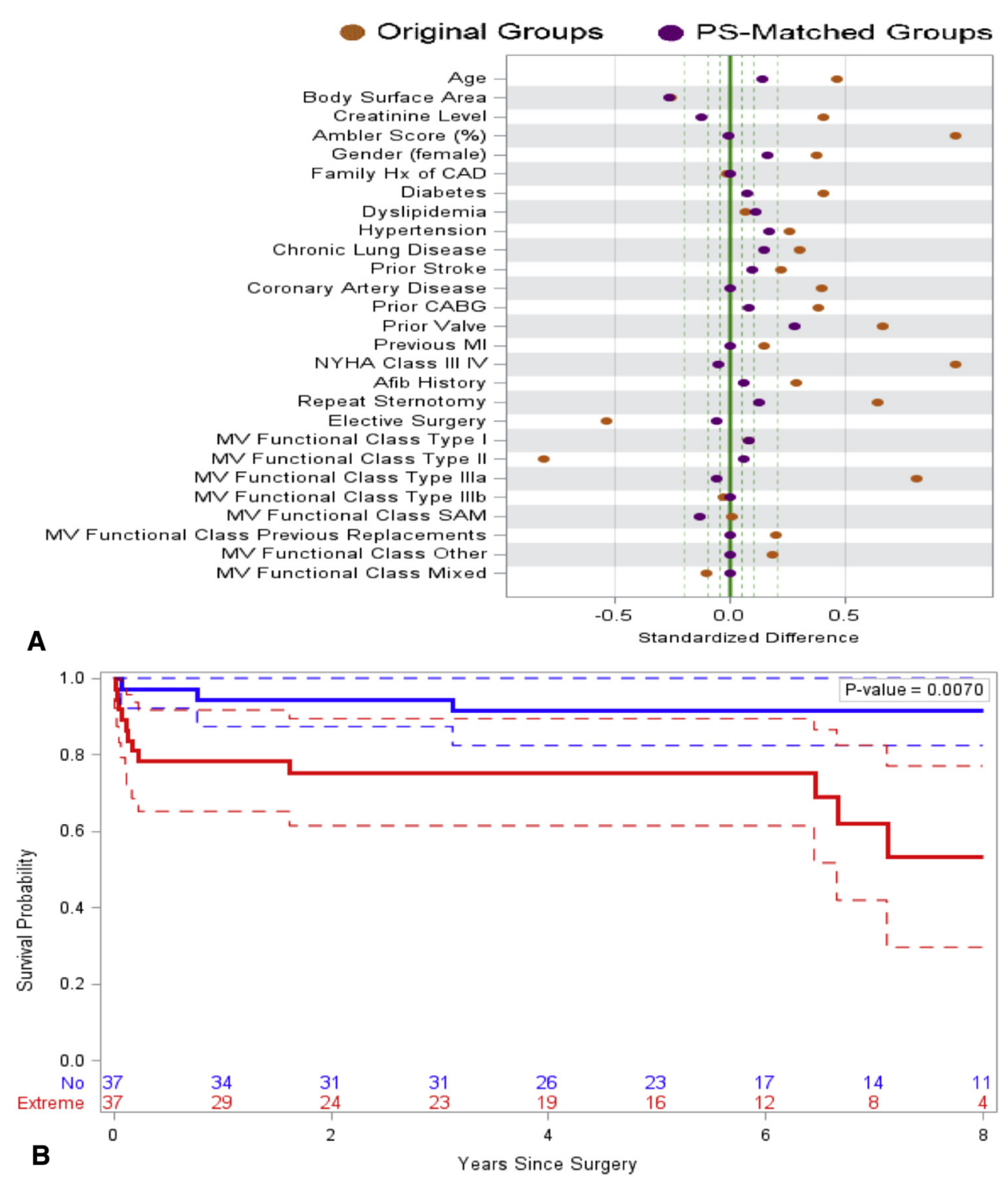

FIGURE 3. Standardized differences in: (A) baseline covariates; and (B) Kaplan-Meier survival estimates in propensity score-matched analyses for extreme versus no PHT. PS, Propensity score; $H x$, history; $C A D$, coronary artery disease; $C A B G$, coronary artery bypass graft; $M I$, myocardial infarction; NYHA, New York Heart Association; Afib, atrial fibrillation; $M V$, mitral valve; SAM, systolic anterior motion.

implantation prior to discharge, compared with those that did not undergo concomitant TVS, before matching $(11.7 \%$ vs $6.1 \%, P=.001)$, but not propensity-score matching $(10.3 \%$ vs $6.7 \%, P=.13)$. After excluding discharge deaths, and patients who had a permanent pacemaker preoperatively, concomitant TVS for repair or replacement was associated with a higher incidence of permanent pacemaker implantation before discharge, in the original groups $(13.6 \%$ vs $6.6 \%, P=.001)$, compared with the propensity-score matched groups $(13.2 \%$ vs $6.8 \%, P=.021)$.

For concomitant TVS for repair, versus no TVS, the incidence of pacemaker implantation before discharge was significantly different in the groups before matching
$(11.2 \%$ vs $6.1 \%$, respectively, $P=.003)$, but not in the propensity-score matched groups $(10.2 \%$ vs $7.1 \%$, $P=.18)$. After excluding discharge deaths and preoperative permanent pacemaker cases, a higher incidence was observed among concomitant TVS for repair patients $(13.5 \%$ vs $6.6 \%, P=.001)$ in the groups before matching, but only a trend was observed propensity-score matching $(12.4 \%$ vs $7.7 \%$, respectively, $P=.09)$.

\section{DISCUSSION}

Propensity-score matched continuous variable, and multivariable analysis, of patients who underwent MVS indicates that severe PHT is not a significant risk factor for short- or long-term unfavorable outcomes. Multiple 

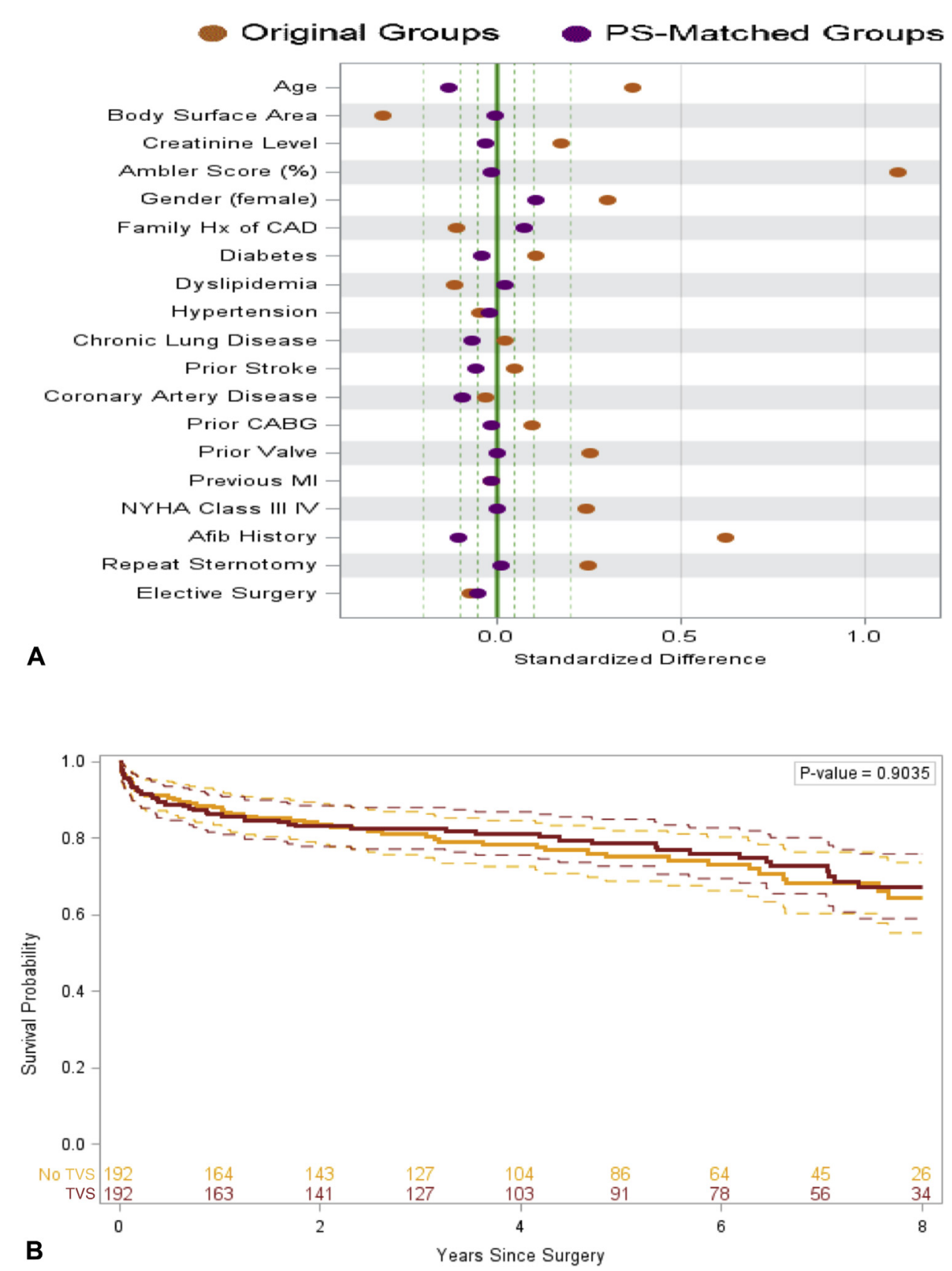

FIGURE 4. Standardized differences in: (A) baseline covariates; and (B) Kaplan-Meier survival estimates in PS-matched analyses by concurrent TVS status in patients with PHT undergoing MVS. $P S$, Propensity score; $H x$, history; $C A D$, coronary artery disease; $C A B G$, coronary artery bypass graft; $M I$, myocardial infarction; NYHA, New York Heart Association; Afib, atrial fibrillation; TVS, tricuspid valve surgery.

prior studies have grouped patients who have PASP $>50 \mathrm{~mm} \mathrm{Hg}$. By separately stratifying patients in the extreme PASP group with those who have PASP $>80 \mathrm{~mm}$ $\mathrm{Hg}$, we have observed comparable outcomes between patients who have severe PHT and those without evidence of PHT. These results suggest that the degree of PHT may be an important consideration when calculating risk for postoperative mortality. Perhaps PHT should only raise reluctance to operate when it is extremely elevated $(>80 \mathrm{~mm} \mathrm{Hg})$, or alternative percutaneous approaches should be pursued. ${ }^{18}$

Prior studies have suggested that PHT adversely affects short- and long-term survival in patients who undergo MVS. ${ }^{19-21}$ The 30-day mortality risk effect of PHT has been included in the EuroSCORE (European System for Cardiac Operative Risk Evaluation) II model (which 


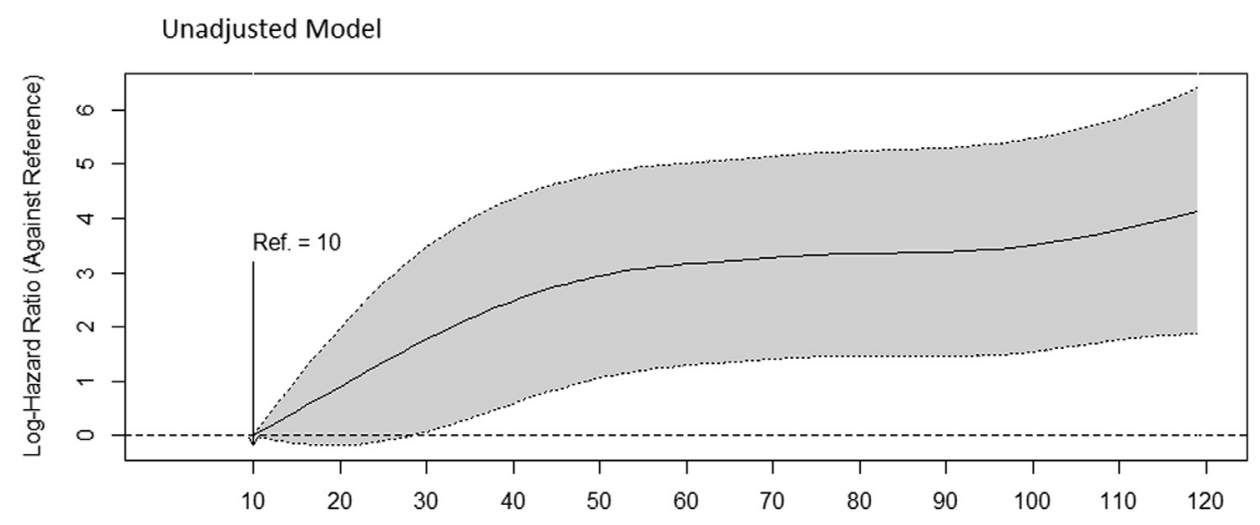

A

Pulmonary Artery Systolic Pressure

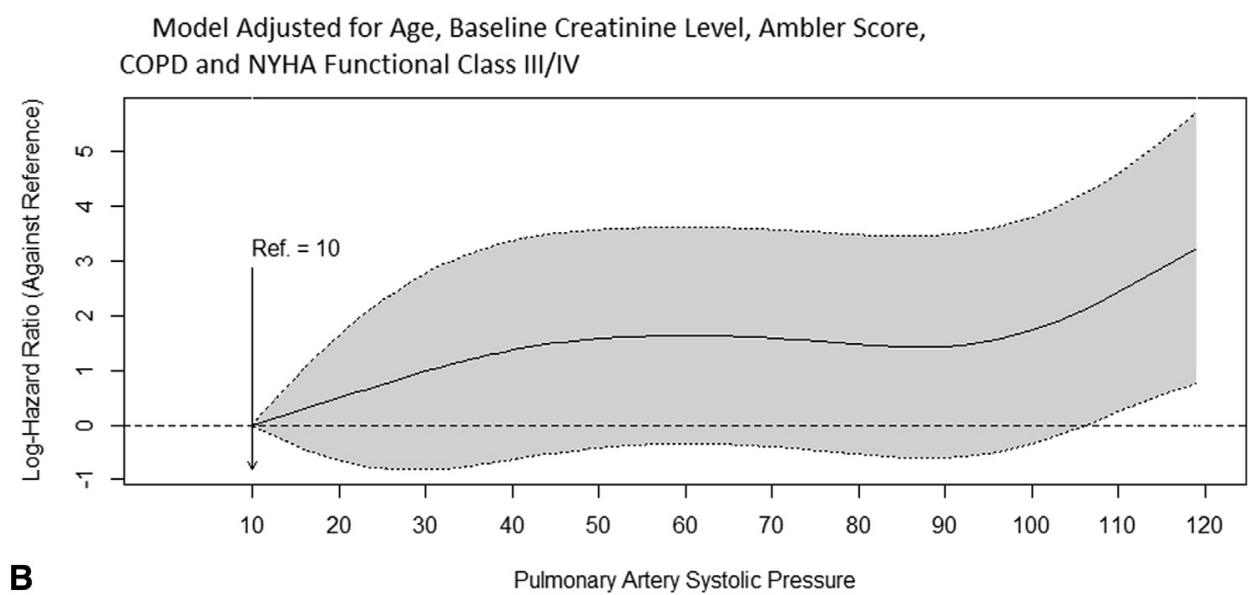

FIGURE 5. Smooth hazard rate estimates for baseline pulmonary artery systolic pressure (as a continuous variable) and corresponding $95 \%$ confidence intervals in (A) unadjusted and (B) covariate-adjusted models. A baseline pulmonary artery systolic pressure value of $10 \mathrm{~mm} \mathrm{Hg}$ serves as the reference point. COPD, Chronic obstructive pulmonary disorder; NYHA, New York Heart Association.

defines PHT as PASP $>30 \mathrm{~mm} \mathrm{Hg}$ ). ${ }^{22}$ The presence of PHT is collected as part of the STS database, but it has not been identified as a mortality risk factor in the STS risk score. ${ }^{23}$ Recent series suggest that PHT should be included in the STS risk score. ${ }^{24}$ However, in multivariate analyses, potential confounders (eg, chronic obstructive pulmonary disorder) in PHT patients undergoing MVS may have exaggerated the mortality risk. ${ }^{25}$ Propensity-score matching can be used to reduce selection bias in nonrandomized trials. ${ }^{26-28}$

Additionally, recent publications have demonstrated increased PHT to be an independent risk factor in both coronary artery bypass grafting and aortic valve replacement. Kennedy and colleagues ${ }^{24}$ reviewed more than 3000 cardiac surgical patients, finding that PHT was significantly associated with increased morbidity and mortality, even after accounting for STS risk. However, mitral operations made up a minor part of their group $(6.9 \%)$, whereas most were coronary artery bypass grafting $(67.5 \%)$ and aortic valve replacement $(24.9 \%)$. Our study focused on MVS exclusively, although 909 of 1571 (57.9\%) patients underwent concomitant revascularization or another valvular procedure. In 2385 patients undergoing primary aortic valve replacement at the Cleveland Clinic, PHT was associated with worse early and late outcomes. In addition, post-aortic valve replacement right ventricular systolic pressure improvement was not stable, and it returned to preoperative levels after 3 to 4 years. ${ }^{29}$ These outcomes suggested that earlier intervention for aortic stenosis could improve outcomes.

As for mitral valve replacement, Ghoreishi and colleagues ${ }^{19}$ reported on the impact of preoperative PHT (defined as PASP $\geq 40 \mathrm{~mm} \mathrm{Hg}$ ) in surgical patients who had mitral regurgitation $(n=888)$. Most of their patients underwent RHC $(68 \%)$. Using multivariate analysis, they concluded that preoperative PASP was a predictor of late death (odds ratio: 1.018 per $1 \mathrm{~mm} \mathrm{Hg}$ increase; 95\% confidence interval: $1.007-1.028 ; P=.001$ ). Their analysis yielded a hospital mortality of $12 \%$ in the severe group (PASP $>60 \mathrm{~mm} \mathrm{Hg}$ ), similar to our mortality result of 6 of $49(12 \%)$ in the extreme group (>80 mm Hg). They concluded that referral for MVS should be considered for 
TABLE 3. Incidence of predischarge permanent pacemaker implantation, by concomitant TVS status

\begin{tabular}{|c|c|c|c|c|c|c|c|}
\hline \multirow[b]{3}{*}{ Comparison } & \multirow[b]{3}{*}{ Exclusions } & \multicolumn{6}{|c|}{ Groups } \\
\hline & & \multicolumn{3}{|c|}{ Before matching } & \multicolumn{3}{|c|}{ After propensity-score matching } \\
\hline & & Size (n) & Pacemaker $(\%)$ & $P$ value & Size (n) & Pacemaker $(\%)$ & $P$ value \\
\hline \multirow[t]{2}{*}{ Entire cohort* } & No & 392 vs 1179 & 12.0 vs 4.6 & .001 & 186 vs 186 & 12.4 vs 6.0 & .031 \\
\hline & Yes & 321 vs 1103 & 14.0 vs 4.9 & .001 & 164 vs 164 & 15.2 vs 7.9 & .038 \\
\hline \multirow[t]{2}{*}{ Entire cohort $\dagger$} & No & 378 vs 1179 & 11.4 vs 4.6 & .001 & 327 vs 327 & 10.1 vs 6.7 & .12 \\
\hline & Yes & 315 vs 1103 & 13.7 vs 4.9 & .001 & 262 vs 262 & $13.0 \mathrm{vs} 7.6$ & .044 \\
\hline \multirow[t]{2}{*}{ PHT patients* } & No & 350 vs 725 & 11.7 vs 6.1 & .001 & 282 vs 282 & 10.3 vs 6.7 & .13 \\
\hline & Yes & 286 vs 668 & 13.6 vs 6.6 & .001 & 235 vs 235 & 13.2 vs 6.8 & .021 \\
\hline \multirow[t]{2}{*}{ PHT patients $\dagger$} & No & 339 vs 725 & 11.2 vs 6.1 & .003 & 283 vs 283 & 10.2 vs 7.1 & .18 \\
\hline & Yes & 281 vs 668 & 13.5 vs 6.6 & .001 & 233 vs 233 & 12.4 vs 7.7 & .09 \\
\hline
\end{tabular}

Values are for TVS (*repair or replacement) or (†repair only) versus no TVS. Exclusions were discharge deaths and preoperative permanent pacemaker implantation. Analyses were performed separately in the entire cohort versus in the subgroup of patients who had preoperative PHT. PHT, Pulmonary hypertension.

patients who have PASP $\geq 40 \mathrm{~mm} \mathrm{Hg}$, given the increased surgical risk.

In contrast, our data suggest that surgery can be performed without increased risk from PHT as an independent factor, up to PASP of $80 \mathrm{~mm} \mathrm{Hg}$. Analysis of PASP and a continuous variable indicates that its effect on mortality is not linear and is statistically significant only toward the high end of the spectrum (Figure 5). In contrast, Ghoreishi and colleagues ${ }^{19}$ have assumed a constant PASP effect; hence, the inference can be drawn that a PASP increase from 30 to $40 \mathrm{~mm} \mathrm{Hg}$ has the same effect on mortality as an increase from 80 to $90 \mathrm{~mm} \mathrm{Hg}$. Therefore, their model leads to an averaged PASP effect, but does not capture the differential PASP effect across its entire spectrum.

Tricuspid regurgitation may confound comparisons of patients who undergo MVS and have PHT. Our subgroup analysis of such patients demonstrated no significant differences in short -or long-term mortality, by concomitant TVS status. Our results suggest that when performing MVS on patients who have comorbid tricuspid regurgitation, TVS can be added without additional mortality risk. However, concomitant TVS (repair or replacement) was associated with a higher incidence of permanent pacemaker implantation prior to discharge, compared with no TVS, in both the before-matching and propensity-score matching groups.

\section{Limitations}

Our analysis has several limitations. PASP was obtained using both RHC and echocardiography, without recording the method in our database. For patients who have suspected PHT, however, RHC was often collected. Thus, RHC PASP measurements, considered the gold standard ${ }^{30}$ in accurately measuring pulmonary artery pressures, were more often obtained in the severe- and extreme-risk groups. In addition, even with propensity-score matching, unmeasured characteristics and confounders may be present. ${ }^{31}$ Our results refer to MVS patients specifically, and are thus not applicable to general cardiac surgical patients (for coronary artery bypass grafting, aortic valve surgery, etc). Despite our best efforts, maintaining the most up-to-date mortality status for each patient continues to be challenging. However, our multilayered and inclusive assessment process is designed to guard against selective reporting according to PHT degree of severity or any other potential risk factors.

\section{CONCLUSIONS}

Early and late mortality in MVS are not significantly associated with severe PHT or the addition of TVS, yet extreme PHT is associated with a higher risk of long-term mortality. Severe PHT (PASP 50-79 mm Hg) should not, in itself, cause a patient to be turned down for surgery, and concomitant TVS can be performed with no significant increase in short- or long-term mortality risks.

\section{Conflict of Interest Statement}

S. Chris Malaisrie reports nonfinancial study approval from Edwards. Sanjiv J. Shah reports consulting fees from Novartis, Bayer, AstraZeneca, and DC Devices. James D. Thomas reports personal fees from Edwards, GE, and Abbott. All other authors have nothing to disclose with regard to commercial support.

You can watch a Webcast of this AATS meeting presentation by going to: http://webcast.aats.org/2015/Video/ Monday/04-27-15_4E_1555_Enter.mp4.

\section{References}

1. Galiè N, Hoeper MM, Humbert M, Torbicki A, Vachiery JL, Barbera JA, et al. Guidelines for the diagnosis and treatment of pulmonary hypertension. Eur Resp J. 2009;34:1219-63.

2. Thunberg CA, Gaitan BD, Grewal A, Ramakrishna H, Stansbury LG, Grigore AM. Pulmonary hypertension in patients undergoing cardiac surgery: 
pathophysiology, perioperative management, and outcomes. J Cardiothor Vasc Anesth. 2013;27:551-72.

3. McLaughlin VV, Archer SL, Badesch DB, Barst RJ, Farber HW, Lindner JR, et al. ACCF/AHA 2009 expert consensus document on pulmonary hypertension: a report of the American College of Cardiology Foundation Task Force on Expert Consensus Documents and the American Heart Association, developed in collaboration with the American College of Chest Physicians; American Thoracic Society, Inc.; and the Pulmonary Hypertension Association. J Am Coll Cardiol. 2009;53:1573-619.

4. Magne J, Pibarot P, Sengupta PP, Donal E, Rosenhek R, Lancellotti P. Pulmonary hypertension in valvular disease: a comprehensive review on pathophysiology to therapy from the HAVEC Group. JACC: Cardiovasc Imaging. 2015;8:83-99.

5. Taramasso M, Vanermen H, Maisano F, Guidotti A, La Canna G, Alfieri O. The growing clinical importance of secondary tricuspid regurgitation. J Am Coll Cardiol. 2012;59:703-10.

6. Nishimura RA, Otto CM, Bonow RO, Carabello BA, Erwin JP III, Guyton RA, et al. 2014 AHA/ACC guideline for the management of patients with valvular heart disease: a report of the American College of Cardiology/American Heart Association Task Force on Practice Guidelines. J Thorac Cardiovasc Surg. 2014;148:e1-132.

7. Douglas PS, Garcia MJ, Haines DE, Lai WW, Manning WJ, Patel AR, et al. ACCF/ASE/AHA/ASNC/HFSA/HRS/SCAI/SCCM/SCCT/SCMR 2011 appropriate use criteria for echocardiography: a report of the American College of Cardiology Foundation Appropriate Use Criteria Task Force, American Society of Echocardiography, American Heart Association, American Society of Nuclear Cardiology, Heart Failure Society of America, Heart Rhythm Society, Society for Cardiovascular Angiography and Interventions, Society of Critical Care Medicine, Society of Cardiovascular Computed Tomography, and Society for Cardiovascular Magnetic Resonance Endorsed by the American College of Chest Physicians. J Am Coll Cardiol. 2011;57:1126-66.

8. Shah SJ. Pulmonary hypertension. JAMA. 2012;308:1366-74.

9. Cohen SR, Sell JE, McIntosh CL, Clark RE. Tricuspid regurgitation in patients with acquired, chronic, pure mitral regurgitation. I. Prevalence, diagnosis, and comparison of preoperative clinical and hemodynamic features in patients with and without tricuspid regurgitation. J Thorac Cardiovasc Surg. 1987;94:481-7.

10. De Meester P, De Cock D, Van De Bruaene A, Gabriels C, Buys R, Helsen F, et al. Additional tricuspid annuloplasty in mitral valve surgery results in better clinical outcome. Heart (Br Cardiac Soc). 2015;101:720-6.

11. Nath J, Foster E, Heidenreich PA. Impact of tricuspid regurgitation on long-term survival. J Am Coll Cardiol. 2004;43:405-9.

12. Calafiore AM, Gallina S, Iacò AL, Contini M, Bivona A, Gagliardi M, et al. Mitral valve surgery for functional mitral regurgitation: should moderate-ormore tricuspid regurgitation be treated? A propensity score analysis. Ann Thorac Surg. 2009;87:698-703.

13. Di Mauro M, Bivona A, Iacò AL, Contini M, Gagliardi M, Varone E, et al. Mitral valve surgery for functional mitral regurgitation: prognostic role of tricuspid regurgitation. Eur J Cardio-Thorac Surg. 2009;35:635-40.

14. Navia JL, Brozzi NA, Klein AL, Ling LF, Kittayarak C, Nowicki ER, et al. Moderate tricuspid regurgitation with left-sided degenerative heart valve disease: to repair or not to repair? Ann Thorac Surg. 2012;93:59-69.

15. Galiè N, Hoeper MM, Humbert M, Torbicki A, Vachiery JL, Barbera JA, et al. Guidelines for the diagnosis and treatment of pulmonary hypertension: the Task Force for the Diagnosis and Treatment of Pulmonary Hypertension of the European Society of Cardiology (ESC) and the European Respiratory Society (ERS), endorsed by the International Society of Heart and Lung Transplantation (ISHLT). Eur Heart J. 2009;30:2493-537.

16. Austin PC. Balance diagnostics for comparing the distribution of baseline covariates between treatment groups in propensity-score matched samples. Stat Med. 2009;28:3083-107.

17. Cadarso-Suárez C, Meira-Machado L, Kneib T, Gude F. Flexible hazard ratio curves for continuous predictors in multi-state models: an application to breast cancer data. Stat Model. 2010;10:291-314.

18. Feldman T, Young A. Percutaneous approaches to valve repair for mitral regurgitation. J Am Coll Cardiol. 2014;63:2057-68.

19. Ghoreishi M, Evans CF, DeFilippi CR, Hobbs G, Young CA, Griffith BP, et al. Pulmonary hypertension adversely affects short- and long-term survival after mitral valve operation for mitral regurgitation: implications for timing of surgery. J Thorac Cardiovasc Surg. 2011;142:1439-52.
20. Le Tourneau T, Richardson M, Juthier F, Modine T, Fayad G, Polge AS, et al Echocardiography predictors and prognostic value of pulmonary artery systolic pressure in chronic organic mitral regurgitation. Heart (Br Cardiac Soc). 2010; 96:1311-7.

21. Barbieri A, Bursi F, Grigioni F, Tribouilloy C, Avierinos JF, Michelena HI, et al Prognostic and therapeutic implications of pulmonary hypertension complicating degenerative mitral regurgitation due to flail leaflet: a multicenter long-term international study. Eur Heart J. 2011;32:751-9.

22. Group ES (European System for Cardiac Operative Risk Evaluation) EuroSCORE II Calculator. Available at: http://www.euroscore.org/calc.html. Accessed April 1, 2015.

23. Society of Thoracic Surgeons. STS risk calculator. Available at: http://riskcalc. sts.org/stswebriskcalc/\#/calculate. Accessed April 1, 2015.

24. Kennedy JL, LaPar DJ, Kern JA, Kron IL, Bergin JD, Kamath S, et al. Does the Society of Thoracic Surgeons risk score accurately predict operative mortality for patients with pulmonary hypertension? J Thorac Cardiovasc Surg. 2013;146: 631-7.

25. Chan V, Ahrari A, Ruel M, Elmistekawy E, Hynes M, Mesana TG. Perioperative deaths after mitral valve operations may be overestimated by contemporary risk models. Ann Thorac Surg. 2014;98:605-10.

26. Little RJ, Rubin DB. Causal effects in clinical and epidemiological studies via potential outcomes: concepts and analytical approaches. Annu Rev Publ Health. 2000;21:121-45.

27. Blackstone EH. Comparing apples and oranges. J Thoracic Cardiovasc Surg. 2002; $123: 8-15$.

28. Rubin DB. The design versus the analysis of observational studies for causal effects: parallels with the design of randomized trials. Stat Med. 2007;26:20-36.

29. Roselli EE, Abdel Azim A, Houghtaling PL, Jaber WA, Blackstone EH. Pulmonary hypertension is associated with worse early and late outcomes after aortic valve replacement: implications for transcatheter aortic valve replacement. $J$ Thorac Cardiovasc Surg. 2012;144:1067-74.e2.

30. Fisher MR, Forfia PR, Chamera E, Housten-Harris T, Champion HC, Girgis RE et al. Accuracy of Doppler echocardiography in the hemodynamic assessment of pulmonary hypertension. Am J Resp Crit Care Med. 2009;179:615-21.

31. Joffe MM, Rosenbaum PR. Invited commentary: propensity scores. Am J Epidemiol. 1999;150:327-33.

Key Words: pulmonary hypertension, mitral valve surgery, tricuspid valve surgery

\section{Discussion}

Dr James S. Gammie (Baltimore, Md). Thank you, Dr Girardi, Dr Puskas, and the Association for the opportunity to discuss this manuscript. Dr Enter-thank you for providing me with a copy of the manuscript well in advance. You and your colleagues have performed a retrospective study of a large series of patients undergoing mitral valve operations and have studied the impact of PHT on shortand long-term outcomes. You and your group are to be congratulated on a notably low operative mortality rate of $2.9 \%$.

More than $70 \%$ of your patients had PHT, which you defined as a systolic pulmonary artery pressure $>35 \mathrm{~mm}$ $\mathrm{Hg}$, and you report that this group had more comorbidities, longer and more-complex operations, lower rates of mitral valve repair, and higher perioperative and long-term mortality. You went on to use propensity-score matching to compare outcomes for a matched group of 215 patients, with versus without severe PHT, which you defined as a pulmonary artery pressure of 50 to $69 \mathrm{~mm} \mathrm{Hg}$. Before you did 
the propensity matching, you found that patients in the severe PHT group had an operative mortality 3 times higher than that for those without PHT. After propensity matching, however, you found that severe PHT was not independently associated with short- or long-term survival.

Dr Enter-your presentation was clear and direct and reflects your hard work and strong mentoring of an integrated resident by Dr Pat McCarthy. I have a few questions and one or two comments. Did you consider performing an analysis that excluded the mitral stenosis and the aortic valve replacement population, to more closely understand the impact of PHT on outcomes for patients with isolated mitral regurgitation? As an example, 15\% of your patients had mitral stenosis, which is a pathophysiologically different disease, with a different impact of PHT on outcomes.

Dr Daniel H. Enter (Chicago, Ill). Thank you, Dr Gammie. It is an honor to have you review our work. As for mitral stenosis, $5 \%$ of the patients in this series had pure mitral stenosis, and $10 \%$ had mixed-mitral regurgitation and mitral stenosis. We did do an analysis excluding those $15 \%$ and found similar results: no difference in short- or long-term mortality for patients with versus without PHT.

Dr Gammie. Did you perform a multivariable regression analysis to assess the impact of PHT on short- and long-term outcomes in addition to propensity-score matching?

Dr Enter. We did not. We only performed propensityscore matching. This was chosen specifically and on the advice of our statisticians.

Dr Gammie. These data potentially have important implications for recommendations for timing of MVS, and I believe we need to be careful about drawing conclusions based on relatively small numbers, mixed etiologies, and different operations. I am not sure that your group has presented a convincing case that severe PHT is not an important risk factor for bad outcomes.

For example, in the propensity-matched group, 3\% of both the no-PHT and the severe-PHT group suffered 30 -day mortality. Now, 3\% of 215 people is either 6 or 7 patients who died in each group, and that is a pretty small sample size on which to base strong conclusions. It may be that your analysis is underpowered to detect a mortality difference. It would be of interest to analyze pulmonary artery pressure as a continuous variable and determine what the threshold is for impact on outcomes. How do you reconcile these results that suggest no impact of PHT with other published studies showing that PHT is a powerful predictor of outcomes?

Dr Enter. This is a very good point. I think in one sense we look at this and say, well, the STS risk score doesn't already include pulmonary hypertension, why might that be? Have they looked at it and found that perhaps it's not as compared with EuroSCORE.

At our center specifically, I know there is a premium placed on myocardial protection. Surgeons have a right ventricular temperature probe, which they monitor closely, although I am not sure why our overall rates of mortality and morbidity were lower than those in other published series.

Dr Gammie. Did you assess right-heart function and evaluate its impact on outcomes?

Dr Enter. We did not, other than pulmonary artery pressures that were presented. Those measures are usually qualitative, and not quantitative, and so we do not put very much faith in them.

Dr Gammie. Do you have any specific intra- or postoperative management strategies for patients who have PHT?

Dr Enter. The standard adjuncts of nitric oxide, milrinone, etc, can be used. But in cases in which patients are able to generate pulmonary artery pressures of 80 or $90 \mathrm{~mm} \mathrm{Hg}$, we probably use them less at our center than they are used across the country.

Dr Gammie. Finally, I would like to emphasize that your finding that pulmonary artery pressures decrease, but do not return to normal, after mitral operation reflects the fact that the presence of PHT, once established, does not completely go away, and that is important to remember, and important as we formulate our guidelines on when to intervene.

Dr Bo Yang (Ann Arbor, Mich). We did a similar study of mitral stenosis patients who had PHT- 2 decades of experience, 317 patients. We had a similar finding for the perioperative mortality and the 30-day mortality. We did not see significant difference among the groups of normal pulmonary artery pressure: mild, moderate, and severe PHT.

However, we did find that the long-term survival in 10 or 12 years was significantly different: The group of combined moderate and severe PHT had a much lower long-term survival. The hazard ratio of death was 3 -fold higher in patients who had moderate-to-severe PHT than in those with normal pulmonary artery pressure-mild PHT. We recommended that, for mitral stenosis patients who had PHT, early surgical intervention should be considered before the patients developed moderate PHT (ie, pulmonary artery pressure $<45 \mathrm{~mm} \mathrm{Hg}$ ).

Dr Enter. Thank you. I appreciate that. If I understand you correctly, you looked at mitral stenosis. We did not look at mitral stenosis specifically, although that comprised $15 \%$ of our series.

Dr Marc Ruel (Ottawa, Ontario, Canada). Dr Enter-a very interesting study, but your results are contrary to so many previous publications in the valve and coronary artery bypass realms, in which it has been shown that PHT is one of the most potent predictors of perioperative mortality and long-term mortality. I think we need to understand why you came to those conclusions.

Essentially, there are 3 types of PHT, 2 of which would be captured in your study as increased systolic pulmonary artery pressures, but 1 of which might not be, and this latter type is 
the patient in whom right ventricular function is so poor that it is actually not generating right ventricular systolic pressures sufficient to be classified as PHT in your study.

Within the first 2 types, ie, arterial and venous with increased measured pressures, presumably the majority of your patients had pulmonary venous hypertension, and one would expect that they would do well, and conversely, that the ones who have pulmonary arterial hypertension would not. Were you able to tease out the subclassifications of PHT so you can enlighten us?

Dr Enter. Thank you for that interesting question. The method of analysis is certainly of import here. Dr Gammie's series, and other very large ones, used multivariate analysis and had a different conclusion from ours, using propensity scoring. So that may be one relevant variable.

As to whether very high pulmonary pressures are the only ones you should use in terms of right ventricular functionthis is an important point - that if your right ventricle is tired out, you might not be able to generate those 80 or $90 \mathrm{~mm} \mathrm{Hg}$ pressures. So it would be interesting to look back at our data and see in the no PHT group is it bimodal in some sense and are patients who can no longer even generate those high pressures. It is not something that we have done thus far, but I would be interested in doing that evaluation.

Dr John D. Puskas (New York, NY). This morning, in this session, we had a vigorous discussion of tricuspid valve repair at the time of mitral repair. What were the indications for tricuspid valve repair in this study? Was it moderate or severe or mild tricuspid regurgitation or just annular dilation? Why do you think that the patients with significant PHT who had versus did not have tricuspid repair had identical 30-day and 5-year survival?
Dr Enter. Thank you for that question. The indications at our center clearly vary among the individual surgeons but follow the guidelines of moderate-to-severe tricuspid regurgitation in the setting of severe mitral regurgitation, and then PHT and annular dilation are factors in addition. I am not sure why the mortality was no different, to be honest with you.

Dr Puskas. One might have anticipated a benefit of tricuspid repair in patients who had PHT who survived their surgery. One might expect those curves to diverge at 5 years - the curves among survivors who had tricuspid repair versus those who did not have tricuspid repair—but they did not. That seems counterintuitive.

Dr Enter. Those patients were sicker patients who required the repair initially, and so that plays a role in our unmatched data. In the matched data, they have similar outcomes. I agree, we could have expected mortality differences to arise in the long-term data if tricuspid repair provided benefit.

Dr Gammie. Vinay Badhwar presented the STS data at the Conclave, and in multivariable analysis, the addition of tricuspid valve repair did not increase mortality. In fact, there was a trend toward lower mortality.

Dr Puskas. No, I would have expected a decreased mortality with repair, but we did not see that. That is what I am surprised at.

Dr Gammie. But it did not seem to adversely affect mortality, fixing the tricuspid valve. I would just mention that the vigorous discussion this morning is just another bolster and support for us to carry out a prospective randomized trial to understand in which specific situations we should be fixing the tricuspid valve.

Dr Puskas. I couldn't agree more. 